\title{
The Use of Green Fluorescent Protein-Tagged Recombinant Viruses to Test Lettuce mosaic virus Resistance in Lettuce
}

\author{
T. Candresse, O. Le Gall, B. Maisonneuve, S. German-Retana, and E. Redondo
}

First, second, fourth, and fifth authors: Equipe de Virologie, UMR GD2P, IBVM, Centre INRA de Bordeaux, BP81, 33883 Villenave d'Ornon cedex, France; and third author: Unité de Génétique et d'Amélioration des Fruits et Légumes, INRA, Domaine Saint Maurice, BP94, 84143 Montfavet cedex, France. Accepted for publication 4 October 2001.

\section{ABSTRACT}

Candresse, T., Le Gall, O., Maisonneuve, B., German-Retana, S., and Redondo, E. 2002. The use of green fluorescent protein-tagged recombinant viruses to test Lettuce mosaic virus resistance in lettuce. Phytopathology 92:169-176.

Seed certification and the use of cultivars containing one of two, probably allelic, recessive genes, $\mathrm{mol}^{1}$ and $\mathrm{mol}^{2}$, are the principal control methods for Lettuce mosaic virus (LMV) in lettuce. Although for a few LMV isolates, $\mathrm{mol}^{2}$ confers resistance with most isolates, the genes $m o 1^{1}$ or $m o l^{2}$ confer a tolerance, and virus accumulation is readily detected in mol-carrying plants. This phenotype complicates evaluation of the resistance status, in particular for $\mathrm{mol}^{1}$, for which there are no viral strains against which a true resistance is expressed. Two green fluorescent protein (GFP)-tagged viruses were constructed, derived from a non-resistance breaking isolate (LMV-0) and from a resistance-breaking isolate (LMV-E).
An evaluation of 101 cultivars of known status was carried out with these recombinant viruses. Using the LMV-0-derived recombinant, identification of mol-carrying cultivars was simple because, contrary to its wild-type parent, systemic movement of LMV-0-GFP was abolished in resistant plants. This assay detected four cases of misidentification of resistance status. In all these cases, further tests confirmed that the prior resistance status information was incorrect, so that a $100 \%$ correlation was observed between LMV-0-GFP behavior and the mol resistance status. Similarly, the LMV-E-derived recombinant allowed the identification of $\mathrm{mol}^{2}$ lettuce lines because its systemic movement was restricted in $\mathrm{mol}^{2}$ lines but not in susceptible or in $\mathrm{mol}^{l}$ lines. The tagged viruses were able to systemically invade another host, pea, irrespective of its resistance status against another member of the genus Potyvirus, Pea seed-borne mosaic virus. The use of these recombinant viruses could therefore greatly facilitate LMV resistance evaluation and speed up lettuce breeding programs.
Lettuce mosaic virus (LMV), a member of the genus Potyvirus $(26,27)$, is probably the most detrimental virus on lettuce crops worldwide (3). The virus is currently controlled by two distinct approaches: the use of virus-free seeds $(5,8)$ supplemented in California with weed control measures and with an annual lettucefree period of 2 weeks or, in Europe, by cultivation of resistant cultivars $(3,29)$. A dominant gene, $M o 2$, providing immunity to LMV infection has been identified in a few lettuce cultivars (15). However, this gene is not useful in breeding programs because it is ineffective against the majority of LMV isolates $(2,3,18)$. The only two resistance genes currently used to protect lettuce crops worldwide are therefore two recessive genes, $\mathrm{mol}^{1}$ and $m o l^{2}$. The $m o l^{l}$ gene, formerly named $g$, was initially identified in cv. Gallega de Invierno $(1,28)$. It was mostly used by European breeders and has now been introduced into all types of lettuce, including butterhead, batavia, looseleaf, crisphead, and cos lettuce (15). The $m o l^{2}$ gene identified in PI 251245, an accession of Lactuca sativa from Egypt $(20,21)$ and originally named mo, has mostly been used by North American breeders who introduced it into crisphead and cos types of lettuce (15). Initially considered identical (20), these genes were later shown to have different specificities and to be either very closely linked or allelic genes and therefore were renamed $\mathrm{mol}^{1}$ and $\mathrm{mol}^{2}(3,15)$.

Corresponding author: T. Candresse; E-mail address: tc@bordeaux.inra.fr

* The $e$-Xtra logo stands for "electronic extra" and indicates the HTML abstract available on-line contains supplemental material not included in the print edition. Figure 1 is in color on-line.

Publication no. P-2001-1205-01R

(C) 2002 The American Phytopathological Society
In lettuce, LMV causes a variety of symptoms, which include vein clearing, mosaic, mottling or necrosis of the leaves, leaf deformation, dwarfing, and defective heading (3). Symptoms are quite variable and depend on the cultivar, the environmental conditions, and the developmental stage at which the plant became infected. Although symptoms on leaves are usually easy to detect, they may be much less conspicuous on some plant types such as varieties containing anthocyanin pigments or batavia-type cultivars with light green and irregular leaves (3). For a few LMV isolates such as LMV-1 or LMV-9, which overcome $m o l^{l}$, the $m o l^{2}$ allele confers a true resistance with no detectable virus accumulation in the resistant cultivars $(2,3,18)$. However, with most isolates, such as LMV-0, $m o l^{l}$ and $m o l^{2}$ confer only tolerance and virus accumulation is usually observed in varieties bearing these genes $(2,3,10,12,18,29)$. These genes can thus be considered both as resistance and tolerance genes, depending on the viral isolate to which they are confronted. The common usage is, however, to use the term resistance to describe them and we have kept this wording throughout this work, despite the fact that the term tolerance would be more appropriate to describe the interaction under most circumstances. Although the viral concentrations reached in molcarrying varieties may be lower than that observed in susceptible ones $(14,15)$, this effect is not observed in some virus-cultivar combinations $(2,18,29)$. As a result, with the exception of the use of LMV-1 or LMV-9 in $\mathrm{mol}^{2}$ cultivars, enzyme-linked immunosorbent assay (ELISA) is not an efficient way to determine the resistance status, and visual assessment of symptoms may be a more effective way of distinguishing resistant from susceptible plants (10). However, depending on the genetic background and the particular resistance gene present, the symptoms induced on resistant cultivars by common strains of LMV may vary from no symptoms at all to some, usually faint, level of chlorosis, mottling, or blotching $(2,3,10,15,18,29)$. 
Because the symptom protection afforded by the resistance genes is partially incomplete and affected by both varietal type and environmental conditions, evaluation of resistance on the basis of symptomatology is often difficult to perform (10), requiring either field experiments (29) or repeated observations under controlled conditions in growth chambers $(2,18)$. As a consequence, and as is also the case for incomplete recessive resistance to other potyviruses (9), evaluation of resistance to LMV can yield confusing results. For example, misidentification of the resistance status of some plants or inappropriate interpretation of segregation data may explain why, using some of the same mapping populations, Montesclaros et al. (12) were not able to map the $m o l^{2}$ gene and concluded that resistance was probably controlled by more than one gene, whereas Irwin et al. (10) mapped the resistance unambiguously to a single locus. Studies on the resistance of cucumber to potyviruses, which show some of the same characteristics, have also resulted in differences in interpretation of the resistance status of cucumber lines or of the dominance status of resistance genes (9). Discrimination between the two resistance alleles $m o l^{1}$ and $\mathrm{mol}^{2}$ in lettuce is also complex and can currently only be achieved by inoculation of $m o l^{l}$-breaking isolates such as LMV-1 or LMV-9 $(15,18)$.

In this study, we report on the use of a green fluorescent protein (GFP) gene-tagged LMV recombinant isolate (LMV-0-GFP) to facilitate the screening of lettuce plants for LMV resistance and the use of a similarly tagged, resistance-breaking isolate (LMV-EGFP) for the identification of the resistance alleles present in a particular variety.

\section{MATERIALS AND METHODS}

Plant materials. Seeds of lettuce cultivars were obtained from the collection of B. Maisonneuve or were provided either by commercial companies or by E. J. Ryder (USDA, Salinas, CA). All experiments were performed in a standard glasshouse (with additional light from $400 \mathrm{~W}$ sodium vapor pressure lamps) and in conformity with the requirements of the French Commission du Génie Génétique for the confinement of recombinant viruses. Lettuce seeds were germinated in sterilized soil and transplanted into 6-cm square pots. Plants were watered by subirrigation and received a fertilizer solution weekly.

Seeds of pea cultivars or of plant introduction lines (PI) were provided by E. Johansen (Danish Institute of Agricultural Sciences, Fredericksburg, Denmark). They were grown and inoculated essentially as described above for the lettuce plants.

Virus isolates and recombinant viruses. Viruses were propagated in plants of susceptible cv. Trocadéro. The common LMV-0 (non-resistance breaking) $(3,18,19)$ and the mol $^{1}$-breaking isolates LMV-1 and LMV-9 $(3,18)$ were used to verify the resistance status of 13 cultivars.

The GFP-tagged recombinant LMV-E isolate (LMV-E-GFP) used in this study has previously been described (7). This recombinant was derived from LMV-E; an isolate able to overcome the resistance conferred by both the $\mathrm{mol}^{1}$ and the $\mathrm{mol}^{2}$ resistance genes $(3,15,18,19)$. The GFP was inserted in frame in the viral polyprotein and is expressed as a translational fusion to the viral helper component (HC-Pro) protein.

A similar recombinant (LMV-0-GFP) was prepared from an infectious cDNA clone of LMV-0 $(15,18,19,31)$ by inserting the GFP gene in the same manner in the viral genome. Both recombinant viruses were initially inoculated in cDNA form to plants of susceptible cv. Trocadéro by particle bombardment (31) and were later propagated in virus form by monthly passages in the same variety.

Virus propagation and resistance assays. Viral isolates were mechanically propagated as previously described $(7,18)$ by rubbing two developed leaves on each plant with a suspension obtained by grinding $1 \mathrm{~g}$ of infected symptomatic lettuce leaves in $5 \mathrm{ml}$ of inoculation buffer $\left(50 \mathrm{mM} \mathrm{Na} \mathrm{HPO}_{4}\right.$ and $0.2 \%$ sodium diethyldithiocarbamate) and adding $90 \mathrm{mg} / \mathrm{ml}$ of activated charcoal and $90 \mathrm{mg} / \mathrm{ml}$ of Carborundum. Following mechanical inoculation, plants were briefly rinsed with tap water. Usually four plants were inoculated per variety for the recombinant virus resistance assays and three plants for the natural viral isolates inoculations.

Plants were observed at frequent intervals for symptom development. GFP fluorescence was detected in a dark room or in the greenhouse at night with a $100 \mathrm{~W}$ hand-held long wave UV spotlight (Model B-100; UV Products, Upland, CA) (7).

\section{RESULTS}

Use of LMV-0-GFP in LMV resistance assays. In a preliminary experiment, the recombinant LMV-0 isolate expressing GFP as a fusion to HC-Pro (7) was inoculated to a differential set of lettuce cultivars (18). This initial experiment showed that LMV-0GFP readily invaded susceptible cultivars such as 'Trocadéro' and could readily be detected in these plants by the high level of GFP fluorescence expressed (Fig. 1A and B). In contrast, no fluorescence was observed on leaves of three resistant cultivars, one harboring $\mathrm{mol}^{l}$ ('Mantilia') and the other two $\mathrm{mol}^{2}$ ('Vanguard 75' [22] and 'Salinas 88' [23]) (Fig. 1F). A large assay involving a number of susceptible and resistant $\left(\mathrm{mol}^{1}\right.$ or $\left.\mathrm{mol}^{2}\right)$ lettuce cultivars of different lettuce types, including varieties with green, yellow, and red leaves, was therefore performed to determine if inoculation with LMV-0-GFP could be used to differentiate susceptible and resistant lettuce cultivars.

In total, 101 cultivars of known resistance status were inoculated with LMV-0-GFP in seven successive independent experiments. Some cultivars were only tested once, whereas others were assayed in two or three different experiments. All inoculations and fluorescence notations were performed as blind tests under code numbers. The varieties tested included 48 butterhead cultivars (23 susceptible and 25 resistant), seven loose-leaf cultivars (five susceptible and two resistant), 29 batavia or crisphead cultivars (9 susceptible and 20 resistant), and 17 cos or Latin-type cultivars (4 susceptible and 13 resistant). The results obtained are presented in Table 1, and representative pictures illustrating the accumulation of GFP are presented in Figure 1.

Generally speaking, an excellent correlation was observed between the known resistance status of the cultivars and the detection of GFP fluorescence on the upper leaves of the inoculated plants. As reported previously for LMV-E-GFP (7), systemic invasion of the plants was rapid, with a timing comparable to that observed for wild-type virus. As a consequence, the values shown in Table 1 are derived from notations performed 6 to 9 days postinoculation (dpi), with the exception of experiment 5 for which notation was at $18 \mathrm{dpi}$. The notations (presence or absence of fluorescence on upper noninoculated leaves) were changed by observations performed at later times in only three cases out of a total of approximately 500 inoculated plants. These include observation of fluorescence on one susceptible 'Trocadéro' plant at 11 dpi but not at $8 \mathrm{dpi}$ (experiment 4), and observation at $19 \mathrm{dpi}$ (but not at $12 \mathrm{dpi}$, experiment 2) and at $11 \mathrm{dpi}$ (but not at $8 \mathrm{dpi}$, experiment 4) of very weak and localized fluorescence on one or two upper leaves each of resistant cvs. Titan (Table 1) and Krizet (Table 1; Fig. 1E).

Fast systemic movement of LMV-0-GFP occurred in 39 of the 40 known susceptible cultivars studied, and GFP fluorescence was readily detected with a simple hand-held UV spotlight. Usually, all three or four tested plants showed the same behavior, but in two of the experiments (experiments 3 and 4), one (in two cases two) of the four inoculated plants did not show GFP accumulation for 10 cultivars from different types. Because in all cases in which these cultivars were tested twice a homogeneous behavior was observed in the other experiment, these plants most likely represent escapes from the inoculation procedure. 

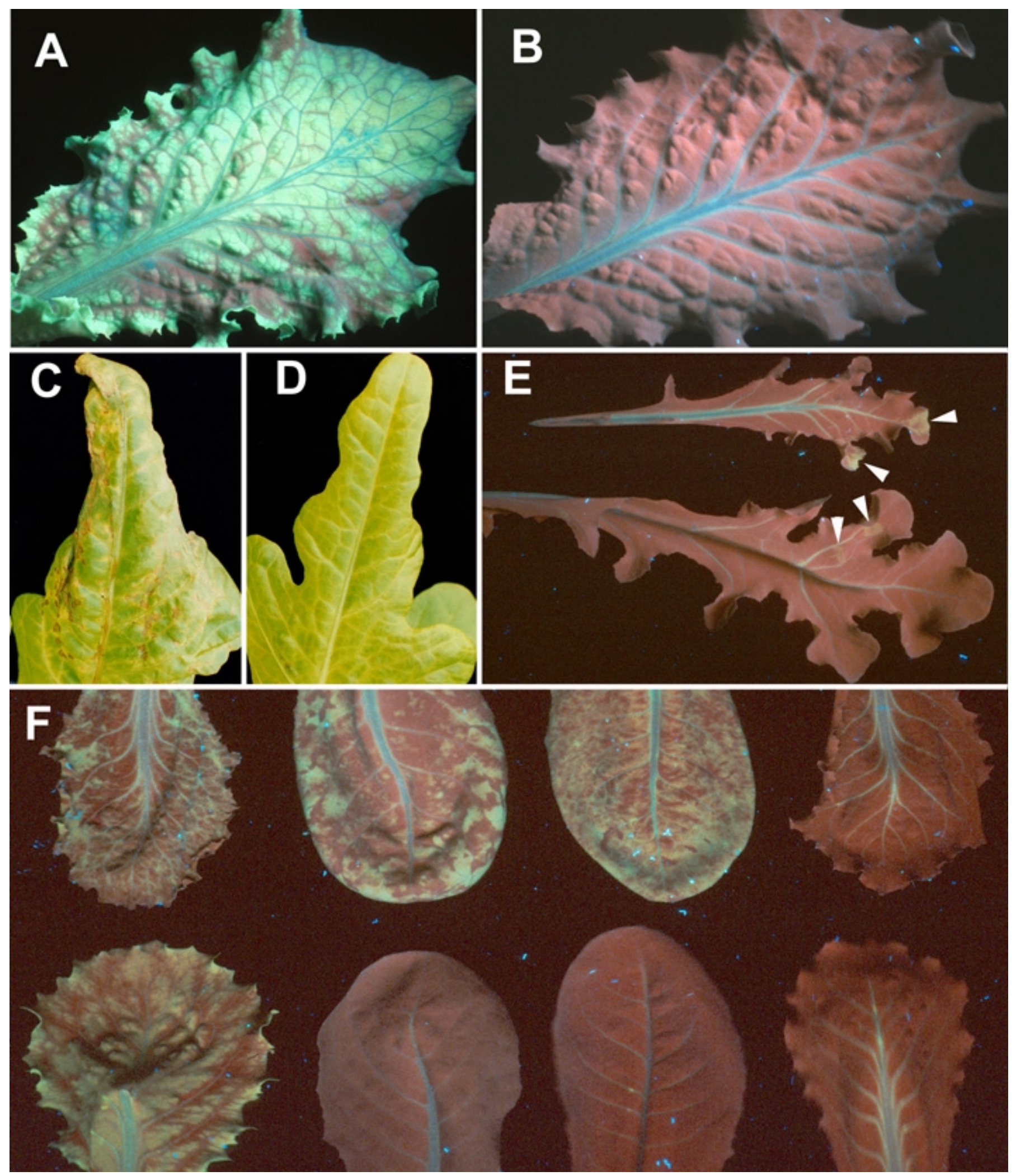

Fig. 1. Symptoms and systemic green fluorescent protein (GFP) accumulation upon inoculation of lettuce plants with recombinant Lettuce mosaic virus (LMV) isolates. A, Accumulation of GFP in a noninoculated leaf of butterhead cv. Angie upon inoculation of LMV-0-GFP. The GFP is green fluorescence on the red background resulting from chlorophyll autofluorescence. Picture taken 14 days postinoculation. B, Healthy control leaf of cv. Angie observed under identical conditions as for A. C, Necrotic symptoms induced in cv. Brunia by LMV-0-GFP. Picture taken 10 days postinoculation. D, Veinclearing symptoms induced in cv. Brunia by nonrecombinant LMV-0. Picture taken 10 days postinoculation. E, Weak GFP fluorescence detected in two leaves of a plant of cv. Krizet (arrows) 14 days after inoculation with LMV-0-GFP (Table 1). F, Detection of GFP accumulation in leaves of lettuce cultivars with different resistance status upon inoculation of LMV-E-GFP (top row) or of LMV-0-GFP (bottom row). The cultivars used from left to right: 'Trocadéro' (susceptible), 'Mantilia' (mo1 ${ }^{I}$ resistant), 'Floribibb' ( $\mathrm{mol}^{1}$ resistant), and 'Vanguard 75' ( $\mathrm{mol}^{2}$ resistant). Picture taken 12 days after inoculation. 
For the susceptible batavia lettuce, in which no systemic accumulation of GFP was observed (variety coded A, Table 1), further tests were performed with the non-resistance breaking isolate LMV-0 (18). The absence of symptoms indicated a wrongly determined resistance status and showed this cultivar to be in fact resistant to LMV (Table 1).

In the 60 known resistant cultivars, systemic accumulation of GFP was only observed in five cases. This included the late and very localized GFP detection in one leaf of one plant each of cvs. Titan (Table 1) and Krizet (Table 1; Fig. 1E) mentioned previously and the fast and effective accumulation of GFP observed in all tested plants of crisphead cv. Fame (Table 1) and cos cvs. Floricrisp and Marquis (Table 1). For these last three cultivars, full susceptibility to LMV infection was confirmed by inoculation with LMV-0 (Table 1).

A complete correlation between the LMV resistance status and the behavior of the LMV-0-GFP recombinant virus was observed (Fig. 1F). Consequently, inoculation with this GFP-tagged recombinant virus derived from LMV-0 provided a fast, simple and reliable way to evaluate the resistance of lettuce cultivars to LMV.

TABLE 1. Systemic green fluorescent protein (GFP) accumulation and Lettuce mosaic virus (LMV)-0 infection symptoms in butterhead lettuce cultivars and leaf, batavia and crisphead, and cos and Latin-type lettuce cultivars inoculated with LMV-0-GFP or LMV-0

\begin{tabular}{|c|c|c|c|c|c|c|c|c|c|c|}
\hline \multirow[b]{2}{*}{ Cultivar } & \multirow[b]{2}{*}{ Company $^{\mathrm{a}}$} & \multirow[b]{2}{*}{ R status ${ }^{\mathrm{b}}$} & \multicolumn{7}{|c|}{ LMV-0-GFPc } & \multirow[b]{2}{*}{ LMV-0 ${ }^{\mathrm{d}}$} \\
\hline & & & 1 & 2 & 3 & 4 & 5 & 6 & 7 & \\
\hline \multicolumn{11}{|c|}{ Butterhead lettuce } \\
\hline Alès & Vilmorin & $\mathrm{S}$ & $\ldots$ & $4 / 4$ & $\ldots$ & $\ldots$ & $\ldots$ & $\ldots$ & $3 / 3$ & $3 / 3$ \\
\hline Ariane & Rijk Zwaan & $\mathrm{S}$ & $\ldots$ & $\ldots$ & $3 / 4$ & $\ldots$ & $\ldots$ & $\ldots$ & $3 / 3$ & $3 / 3$ \\
\hline Columbus & Tézier & $\mathrm{S}$ & $\ldots$ & $\ldots$ & $4 / 4$ & $\ldots$ & $\ldots$ & $\ldots$ & $3 / 3$ & $3 / 3$ \\
\hline Fiona & Gautier & $\mathrm{S}$ & $\ldots$ & $\ldots$ & $4 / 4$ & $\ldots$ & $\ldots$ & $\ldots$ & $3 / 3$ & $3 / 3$ \\
\hline Johana & $S \& G$ & $\mathrm{~S}$ & $\ldots$ & $\ldots$ & $3 / 4$ & $\ldots$ & $\ldots$ & $\ldots$ & $3 / 3$ & $3 / 3$ \\
\hline Judy & $S \& G$ & S & $4 / 4$ & $\ldots$ & $\ldots$ & $\ldots$ & $\ldots$ & $\ldots$ & $3 / 3$ & $3 / 3$ \\
\hline Magdalena & $S \& G$ & $\mathrm{~S}$ & $\ldots$ & $4 / 4$ & $\ldots$ & $\ldots$ & $\ldots$ & $\ldots$ & $3 / 3$ & $3 / 3$ \\
\hline Manita & Rijk Zwaan & $\mathrm{S}$ & $\ldots$ & $\ldots$ & $3 / 4$ & $\ldots$ & $\ldots$ & $\ldots$ & $3 / 3$ & $3 / 3$ \\
\hline Mariska & Numhens & $\mathrm{S}$ & $4 / 4$ & $\ldots$ & $\ldots$ & $\ldots$ & $\ldots$ & $\ldots$ & $3 / 3$ & $3 / 3$ \\
\hline Méliès & Vilmorin & $\mathrm{S}$ & $\ldots$ & $\ldots$ & $3 / 4$ & $4 / 4$ & $\ldots$ & $\ldots$ & $3 / 3$ & $3 / 3$ \\
\hline Ninja & S \& G & S & $\ldots$ & $\ldots$ & $3 / 4$ & $\ldots$ & $\ldots$ & $\ldots$ & $3 / 3$ & $3 / 3$ \\
\hline Sensai & Rijk Zwaan & $\mathrm{S}$ & $\ldots$ & $\ldots$ & $2 / 4$ & $\ldots$ & $\ldots$ & $\ldots$ & $3 / 3$ & $3 / 3$ \\
\hline Trocadéro & Old variety & S & $\ldots$ & $\ldots$ & $\ldots$ & $3 / 4\left(4 / 4^{\mathrm{e}}\right)$ & $\ldots$ & $\ldots$ & $3 / 3$ & $3 / 3$ \\
\hline Tzigane & Rijk Zwaan & $\mathrm{S}$ & $\ldots$ & $4 / 4$ & $\ldots$ & $\ldots$ & $\ldots$ & $\ldots$ & $3 / 3$ & $3 / 3$ \\
\hline Verpia & Clause & $\mathrm{S}$ & $\ldots$ & $\ldots$ & $4 / 4$ & $\ldots$ & $\ldots$ & $\ldots$ & $3 / 3$ & $3 / 3$ \\
\hline Alizé & Gautier & $\mathrm{R}$ & $\ldots$ & $\ldots$ & $0 / 4$ & $0 / 4$ & $\ldots$ & $\ldots$ & $\ldots$ & $\ldots$ \\
\hline Alto & Rijk Zwaan & $\mathrm{R}$ & $\ldots$ & $0 / 4$ & $\ldots$ & $0 / 4$ & $\ldots$ & $\ldots$ & $\ldots$ & $\ldots$ \\
\hline Audran & $S \& G$ & $\mathrm{R}$ & $0 / 4$ & $\ldots$ & $\ldots$ & $\ldots$ & $\ldots$ & $\ldots$ & $\ldots$ & $\ldots$ \\
\hline Augusta & Clause & $\mathrm{R}$ & $\ldots$ & $0 / 4$ & $0 / 4$ & $\ldots$ & $\ldots$ & $\ldots$ & $\ldots$ & $0 / 3$ \\
\hline Daguan & $S \& G$ & $\mathrm{R}$ & $\ldots$ & $0 / 4$ & $\ldots$ & $\ldots$ & $\ldots$ & $\ldots$ & $\ldots$ & $0 / 3$ \\
\hline Divina & Vilmorin & $\mathrm{R}$ & $\ldots$ & $0 / 4$ & $\ldots$ & $\ldots$ & $\ldots$ & $\ldots$ & $\ldots$ & $\ldots$ \\
\hline Enrica & $S \& G$ & $\mathrm{R}$ & $\ldots$ & $0 / 4$ & $\ldots$ & $\ldots$ & $\ldots$ & $\ldots$ & $\ldots$ & $\ldots$ \\
\hline Ermosa & Gautier & $\mathrm{R}$ & $\ldots$ & $0 / 4$ & $\ldots$ & $\ldots$ & $\ldots$ & $\ldots$ & $\ldots$ & $0 / 3$ \\
\hline Locness & Vilmorin & $\mathrm{R}$ & $\ldots$ & $0 / 4$ & $\ldots$ & $\ldots$ & $\ldots$ & $\ldots$ & $\ldots$ & $\ldots$ \\
\hline Lutine & Tézier & $\mathrm{R}$ & $\ldots$ & $0 / 4$ & $\ldots$ & $\ldots$ & $\ldots$ & $\ldots$ & $\ldots$ & $\ldots$ \\
\hline \multirow[t]{2}{*}{ Salad Bibb } & Harris Moran & $\mathrm{R}$ & $\ldots$ & $\ldots$ & $\ldots$ & $\ldots$ & $0 / 3$ & $\cdots$ & $\cdots$ & $\cdots$ \\
\hline & & & & & & & & & \multicolumn{2}{|c|}{ (continued on next page) } \\
\hline
\end{tabular}

a Vilmorin (La Ménitre, France), FAES (Florida Agricultural Experimental Station (Belle Glade), Rijk Zwaan (Aramon, France), Tézier (Portes les Valence, France), Gautier (Graines Gautier, Eyragues, France), S \& G (Sluis \& Groot, Les Ponts de Ce, France), Numhems (Semences Numhens, Sucelles, France), Clause (Brétigny sur Orge, France), INRA (Institut National de la Recherche Agronomique, Avignon, France), Harris Moran (Modesto, CA), Enza Zaden (Allones, France), USDA (U.S. Department of Agriculture, Salinas, CA), Asgrow (Garons, France), Arco (Newton Square, PA), Ferry Morse (Fulton, KY), and Petoseed (Garons, France).

${ }^{\mathrm{b}}$ Reported resistance status: $\mathrm{S}=$ susceptible; $\mathrm{R}=$ resistant.

${ }^{c}$ Number of plants showing systemic GFP accumulation/number of LMV-0-GFP-inoculated plants. Six independent experiments were performed and are indicated by 1 to 5 and 7. Notations were performed 6 (experiment 2), 8 (experiments 4 and 6), 9 (experiments 1, 3, and 7), and 18 days (experiment 5) after inoculation.

${ }^{\mathrm{d}}$ Number of plants with symptoms at 6, 8, or 9 days after inoculation/number of LMV-0-inoculated plants.

e One plant showing GFP accumulation 11 but not 8 days after inoculation.

${ }^{\mathrm{f}}$ One plant showing low GFP accumulation on one leaf 19 days after inoculation.

$\mathrm{g}$ Very severe and necrotic symptoms.

${ }^{\text {h }}$ Plant showing very low GFP accumulation on one leaf 11 days after inoculation (Fig. 1E). 
As previously reported for LMV-E-GFP (7), the symptoms induced by LMV-0-GFP were significantly milder than those induced by the parental LMV-0 isolate (data not shown). This was true for all varietal types and cultivars tested with a single notable exception. In looseleaf cv. Brunia, symptoms induced by LMV-0GFP were considerably stronger than the mosaic induced by LMV-0 (Fig. 1C versus D). These symptoms included very severe stunting and leaf deformation as well as extensive necrosis (Fig. 1C). Necrotic lesions were also frequently observed on inoculated leaves. The same pattern was observed again when 'Brunia' was inoculated with LMV-E-GFP and LMV-E: symptoms induced by the GFP-tagged recombinant were more severe and strongly necrotic compared with the symptoms induced by the nonrecombinant parent (data not shown). This peculiar infection phenotype therefore appears to be linked to the tagging of the HC-Pro with GFP, a fact that was confirmed by the observation that neither LMV-0-GUS nor LMV-E-GUS (7) shows this increased severity phenotype in 'Brunia' (data not shown).

TABLE 1. (continued from preceding page)

\begin{tabular}{|c|c|c|c|c|c|c|c|c|c|c|}
\hline \multirow[b]{2}{*}{ Cultivar } & \multirow[b]{2}{*}{ Company ${ }^{\mathrm{a}}$} & \multirow[b]{2}{*}{ R status ${ }^{b}$} & \multicolumn{7}{|c|}{ LMV-0-GFPc } & \multirow[b]{2}{*}{ LMV-0 } \\
\hline & & & 1 & 2 & 3 & 4 & 5 & 6 & 7 & \\
\hline Sangria & Vilmorin & $\mathrm{R}$ & $\ldots$ & $\ldots$ & $0 / 4$ & $\ldots$ & $\ldots$ & $\ldots$ & $\ldots$ & $\ldots$ \\
\hline Titan & $S \& G$ & $\mathrm{R}$ & $\ldots$ & $0 / 4\left(1 / 4^{f}\right)$ & $\ldots$ & $\ldots$ & $\ldots$ & $\ldots$ & $\ldots$ & $0 / 3$ \\
\hline Verian & $S \& G$ & $\mathrm{R}$ & $\ldots$ & $0 / 4$ & $\ldots$ & $\ldots$ & $\ldots$ & $\ldots$ & $\ldots$ & $\ldots$ \\
\hline F5 line in selection & INRA & $\mathrm{R}$ & $\ldots$ & $0 / 4$ & $\ldots$ & $\ldots$ & $\ldots$ & $\ldots$ & $\ldots$ & $\ldots$ \\
\hline F6 line in selection & INRA & $\mathrm{R}$ & $\ldots$ & $0 / 4$ & $\ldots$ & $\ldots$ & $\ldots$ & $\ldots$ & $\ldots$ & $0 / 3$ \\
\hline \multicolumn{11}{|l|}{ Leaf lettuce } \\
\hline Brunia & Vilmorin & $\mathrm{S}$ & $\ldots$ & $8 / 8^{\mathrm{g}}$ & $\ldots$ & $\ldots$ & $\ldots$ & $\ldots$ & $3 / 3$ & $2 / 2$ \\
\hline Cocarde & Gautier & $\mathrm{S}$ & $4 / 4$ & $\ldots$ & $\ldots$ & $\ldots$ & $\ldots$ & $\ldots$ & $1 / 1$ & $1 / 1$ \\
\hline Feuille de chêne blonde & Old variety & $\mathrm{S}$ & $4 / 4$ & $\ldots$ & $\ldots$ & $\ldots$ & $\ldots$ & $\ldots$ & $3 / 3$ & $3 / 3$ \\
\hline Magalie & Rijk Zwaan & $\mathrm{S}$ & $\ldots$ & $4 / 4$ & $\ldots$ & $\ldots$ & $\ldots$ & $\ldots$ & $3 / 3$ & $3 / 3$ \\
\hline Sigla & Enza Zaden & $?$ & $\ldots$ & $4 / 4$ & $\ldots$ & $\ldots$ & $\ldots$ & $4 / 4$ & $\ldots$ & $4 / 7$ \\
\hline Kristine & Rijk Zwaan & $\mathrm{R}$ & $0 / 4$ & $\ldots$ & $\ldots$ & $\ldots$ & $\ldots$ & $\ldots$ & $\ldots$ & $\ldots$ \\
\hline Krizet & Rijk Zwaan & $\mathrm{R}$ & $\cdots$ & $\cdots$ & $0 / 4$ & $0 / 4\left(1 / 4^{\mathrm{h}}\right)$ & $\cdots$ & $\cdots$ & $\cdots$ & $\cdots$ \\
\hline \multicolumn{11}{|l|}{ Batavia and crisp lettuce } \\
\hline Coded A & Gautier & $\mathrm{S}$ & $\ldots$ & $0 / 4$ & $0 / 4$ & $\ldots$ & $\ldots$ & $\ldots$ & $\ldots$ & $0 / 6$ \\
\hline Dorée de printemps & Gautier & $\mathrm{S}$ & $\ldots$ & $\ldots$ & $3 / 4$ & $\ldots$ & $\ldots$ & $\ldots$ & $3 / 3$ & $3 / 3$ \\
\hline Floréal & Rijk Zwaan & $\mathrm{S}$ & $\ldots$ & $\ldots$ & $3 / 4$ & $\ldots$ & $\ldots$ & $\ldots$ & $3 / 3$ & $3 / 3$ \\
\hline Padana & Clause & $S$ & $\ldots$ & $4 / 4$ & $\ldots$ & $\ldots$ & $\ldots$ & $\ldots$ & $\ldots$ & $2 / 2$ \\
\hline Reines des glaces & Old variety & $\mathrm{S}$ & $\ldots$ & $4 / 4$ & $\ldots$ & $\ldots$ & $\ldots$ & $\ldots$ & $3 / 3$ & $3 / 3$ \\
\hline Salinas & USDA & $\mathrm{S}$ & $4 / 4$ & $\ldots$ & $\ldots$ & $2 / 4$ & $\ldots$ & $\ldots$ & $3 / 3$ & $3 / 3$ \\
\hline Salvina & Clause & $\mathrm{S}$ & $\ldots$ & $4 / 4$ & $\ldots$ & $\ldots$ & $\ldots$ & $\ldots$ & $3 / 3$ & $3 / 3$ \\
\hline Vanguard & USDA & $\mathrm{S}$ & $4 / 4$ & $\ldots$ & $\ldots$ & $4 / 4$ & $\ldots$ & $\ldots$ & $3 / 3$ & $3 / 3$ \\
\hline Verdie & Rijk Zwaan & $\mathrm{S}$ & $\ldots$ & $4 / 4$ & $\ldots$ & $\ldots$ & $\ldots$ & $\ldots$ & $3 / 3$ & $3 / 3$ \\
\hline Aida & $S \& G$ & $\mathrm{R}$ & $\ldots$ & $\ldots$ & $0 / 4$ & $0 / 4$ & $\ldots$ & $\ldots$ & $\ldots$ & $\ldots$ \\
\hline Autumn Gold & USDA & $\mathrm{R}$ & $\ldots$ & $\ldots$ & $\ldots$ & $\ldots$ & $0 / 3$ & $\ldots$ & $\ldots$ & $\ldots$ \\
\hline Canasta & $S \& G$ & $\mathrm{R}$ & $0 / 4$ & $\ldots$ & $\ldots$ & $\ldots$ & $\ldots$ & $\ldots$ & $\ldots$ & $\ldots$ \\
\hline Carioca & Clause & $\mathrm{R}$ & $\ldots$ & $\ldots$ & $0 / 4$ & $\ldots$ & $\ldots$ & $\ldots$ & $\ldots$ & $\ldots$ \\
\hline Carmen & Gautier & $\mathrm{R}$ & $\ldots$ & $0 / 4$ & $\ldots$ & $\ldots$ & $\ldots$ & $\ldots$ & $\ldots$ & $0 / 3$ \\
\hline Classic & Asgrow & $\mathrm{R}$ & $\ldots$ & $\ldots$ & $\ldots$ & $\ldots$ & $\ldots$ & $0 / 4$ & $\ldots$ & $0 / 4$ \\
\hline Desert storm & Harris Moran & $\mathrm{R}$ & $\ldots$ & $\ldots$ & $\ldots$ & $\ldots$ & $\ldots$ & $0 / 4$ & $\ldots$ & $0 / 4$ \\
\hline Dorémi & $S \& G$ & $\mathrm{R}$ & $\ldots$ & $0 / 4$ & $\ldots$ & $\ldots$ & $\ldots$ & $\ldots$ & $\ldots$ & $\ldots$ \\
\hline Fame & Asgrow & $\mathrm{R}$ & $\ldots$ & $\ldots$ & $\ldots$ & $\ldots$ & $3 / 3$ & $4 / 4$ & $\ldots$ & $4 / 4$ \\
\hline Kristia & $S \& G$ & $\mathrm{R}$ & $\ldots$ & $0 / 4$ & $\ldots$ & $\ldots$ & $\ldots$ & $\ldots$ & $\ldots$ & $\ldots$ \\
\hline Malika & $\mathrm{S} \& \mathrm{G}$ & $\mathrm{R}$ & $0 / 4$ & $\ldots$ & $\cdots$ & $\cdots$ & $\cdots$ & $\cdots$ & $\cdots$ & $\cdots$ \\
\hline Masaida & $S \& G$ & $\mathrm{R}$ & $0 / 4$ & $\ldots$ & $\ldots$ & $\ldots$ & $\ldots$ & $\ldots$ & $\ldots$ & $\ldots$ \\
\hline Mojave & Harris Moran & $\mathrm{R}$ & $\ldots$ & $\ldots$ & $\ldots$ & & $0 / 3$ & $\ldots$ & $\ldots$ & $\ldots$ \\
\hline Nevada & Vilmorin & $\mathrm{R}$ & $\ldots$ & $0 / 4$ & $0 / 4$ & $\ldots$ & $\ldots$ & $\ldots$ & $\ldots$ & $0 / 3$ \\
\hline Panthéon & Rijk Zwaan & $\mathrm{R}$ & $\ldots$ & $0 / 4$ & $\ldots$ & $\ldots$ & $\ldots$ & $\ldots$ & $\ldots$ & $\ldots$ \\
\hline Rossia & Clause & $\mathrm{R}$ & $\ldots$ & $0 / 4$ & $0 / 4$ & $\ldots$ & $\ldots$ & $\ldots$ & $\ldots$ & $0 / 3$ \\
\hline Salinas 88 & USDA & $\mathrm{R}$ & $0 / 4$ & $\ldots$ & $\ldots$ & $0 / 4$ & $\ldots$ & $\ldots$ & $\ldots$ & $\ldots$ \\
\hline Sierra & Vilmorin & $\mathrm{R}$ & $\ldots$ & $0 / 4$ & $\ldots$ & $\ldots$ & $\ldots$ & $\ldots$ & $\ldots$ & $\ldots$ \\
\hline Vanguard 75 & USDA & $\mathrm{R}$ & $0 / 4$ & $\ldots$ & $\ldots$ & $0 / 4$ & $\ldots$ & $\ldots$ & $\ldots$ & $\ldots$ \\
\hline Winterset & USDA & $\mathrm{R}$ & $\cdots$ & $\cdots$ & $\ldots$ & $\ldots$ & $\cdots$ & $0 / 4$ & $\ldots$ & $0 / 4$ \\
\hline \multicolumn{11}{|l|}{ Cos/Latin lettuce } \\
\hline Little Leprechaun & Arco & $\mathrm{S}$ & $\ldots$ & $4 / 4$ & $\ldots$ & $\ldots$ & $\ldots$ & $\ldots$ & $3 / 3$ & $3 / 3$ \\
\hline Parris Island Cos & Ferry Morse & $\mathrm{S}$ & $\ldots$ & $4 / 4$ & $4 / 4$ & $\ldots$ & $\ldots$ & $\ldots$ & $\ldots$ & $7 / 7$ \\
\hline Remus & Rijk Zwaan & $\mathrm{S}$ & $\ldots$ & $4 / 4$ & $\ldots$ & $4 / 4$ & $\ldots$ & $\ldots$ & $3 / 3$ & $3 / 3$ \\
\hline Ruby & USDA & $\mathrm{S}$ & $\ldots$ & $4 / 4$ & $\ldots$ & $\ldots$ & $\ldots$ & $\ldots$ & $3 / 3$ & $3 / 3$ \\
\hline Augustus & Petoseed & $\mathrm{R}$ & $\ldots$ & $\ldots$ & $\ldots$ & $\ldots$ & $0 / 3$ & $\ldots$ & $\ldots$ & $\ldots$ \\
\hline Floribibb & FAES & $\mathrm{R}$ & $\ldots$ & $\ldots$ & $0 / 4$ & $0 / 4$ & $\ldots$ & $\ldots$ & $\ldots$ & $\ldots$ \\
\hline Floricos & FAES & $\mathrm{R}$ & $\ldots$ & $\ldots$ & $0 / 4$ & $0 / 4$ & $\ldots$ & $\ldots$ & $\ldots$ & $\ldots$ \\
\hline Floricrisp & FAES & $\mathrm{R}$ & $\ldots$ & $4 / 4$ & $4 / 4$ & $\ldots$ & $\ldots$ & $\ldots$ & $\ldots$ & $6 / 6$ \\
\hline Floriglade & FAES & $\mathrm{R}$ & $\ldots$ & $\ldots$ & $0 / 4$ & $0 / 4$ & $\ldots$ & $\ldots$ & $\ldots$ & $\ldots$ \\
\hline Gallega de Invierno & Old variety & $\mathrm{R}$ & $\ldots$ & $\ldots$ & $0 / 8$ & $\ldots$ & $\ldots$ & $\ldots$ & $\ldots$ & $0 / 3$ \\
\hline Marquis & Asgrow & $\mathrm{R}$ & $\ldots$ & $\ldots$ & $\ldots$ & $\ldots$ & $3 / 3$ & $4 / 4$ & $\ldots$ & $4 / 4$ \\
\hline Plato & Petoseed & $\mathrm{R}$ & $\ldots$ & $\ldots$ & $\ldots$ & $\ldots$ & $0 / 3$ & $\ldots$ & $\ldots$ & $\ldots$ \\
\hline Presidio & Asgrow & $\mathrm{R}$ & $\ldots$ & $\ldots$ & $\ldots$ & $\ldots$ & $0 / 3$ & $\ldots$ & $\ldots$ & $\ldots$ \\
\hline Romulus & Petoseed & $\mathrm{R}$ & $\ldots$ & $\ldots$ & $\ldots$ & $\ldots$ & $0 / 3$ & $\ldots$ & $\ldots$ & $\ldots$ \\
\hline Short Guzmaine & FAES & $\mathrm{R}$ & $\ldots$ & $0 / 4$ & $\ldots$ & $0 / 4$ & $\ldots$ & $\ldots$ & $\ldots$ & $\ldots$ \\
\hline F10 line in selection & INRA & $\mathrm{R}$ & $\ldots$ & $\ldots$ & $0 / 4$ & $\ldots$ & $\ldots$ & $\ldots$ & $\ldots$ & $\ldots$ \\
\hline F6 line in selection & INRA & $\mathrm{R}$ & $\ldots$ & $\ldots$ & $0 / 4$ & $0 / 4$ & $\ldots$ & $\ldots$ & $\ldots$ & $\ldots$ \\
\hline
\end{tabular}


Differentiation of $m o 1^{1}$ and $m o 1^{2}$ resistance alleles by LMVE-GFP. LMV-E is a resistance-breaking isolate able to overcome resistance conferred by the $m o l^{l}$ or the $m o l^{2}$ genes $(3,18)$. However, previous results (7) showed that GFP-tagging of this isolate resulted in an almost complete inability of the virus to move systemically in cv. Vanguard 75, which harbors the $\mathrm{mol}^{2}$ resistance gene (22) (Fig. 1F). In fact, although some systemic movement and limited GFP accumulation was erratically and very occasionally observed (7), most 'Vanguard 75' plants inoculated with LMV-E-GFP failed to show GFP accumulation even 1 or 2 months after inoculation (31, this work). However, later tests showed that LMV-E-GFP was able to move readily in plants of cv. Mantilia, carrying the $\mathrm{mol}^{l}$ gene (Fig. 1F). This observation indicated that LMV-E-GFP could not be used to discriminate between resistant and susceptible cultivars but suggested a series of experiments to determine whether LMV-E-GFP could be used to differentiate varieties carrying the $\mathrm{mol}^{l}$ and $\mathrm{mol}^{2}$ resistance genes. The results of these experiments are summarized in Table 2.

In total, 36 cultivars representing different varietal types were used in these experiments. These included three susceptible varieties, $23 \mathrm{~mol}^{1}$, and nine $\mathrm{mol}^{2}$ varieties. The nature of the allele present in cv. Floribibb was unknown because its ancestry contained both $\mathrm{mol}^{l}$ and $\mathrm{mol}^{2}$ carrying lines (E. J. Ryder, personal communication). As for the experiments with LMV-0-GFP reported previously, systemic movement of LMV-E-GFP and
GFP accumulation occurred rapidly and initial observations (performed 8 or 9 days after inoculation) were only very occasionally modified by observations at later times.

In all three susceptible cultivar (as well as in numerous others tested in different experiments, data not shown), systemic movement of LMV-E-GFP and GFP accumulation was readily observed (Fig. $1 \mathrm{~F})$. This was also the case in all $\mathrm{mol}^{\mathrm{l}}$-carrying varieties, although 18 of 228 inoculated plants (nine cultivars) apparently escaped infection (Table 2). The fact that these varieties carry $\mathrm{mol}^{1}$ and not $\mathrm{mol}^{2}$ was confirmed for some of them by inoculation with $\mathrm{mol}^{1}$ breaking LMV isolates LMV-1 or LMV-9 $(3,18)$. The resistance gene carried by cv. Floribibb was indicated as $\mathrm{mol}^{l}$ by the ability of LMV-E-GFP to move systemically in this cultivar (Fig. 1F). This identification was confirmed by the development of symptoms upon inoculation of plants of this variety with LMV-1 (Table 2).

In contrast and similar to what had previously been observed in cv. Vanguard 75 (7), no systemic movement or GFP accumulation was observed in the nine cultivars carrying the $\mathrm{mol}^{2}$ gene, even though some of the plants tested belonged to different varietal types (Table 2; Fig. 1F). Again, the presence of the $\mathrm{mol}^{2}$ allele was confirmed for the varieties for which some doubts existed due to incomplete information (24) by their resistance to the $\mathrm{mol}^{1}$ breaking isolates LMV-1 or LMV-9 (Table 2).

The use of the LMV-E-GFP recombinant virus therefore provided a fast and straightforward way to differentiate lettuce

TABLE 2. Systemic green fluorescent protein (GFP) accumulation and Lettuce mosaic virus (LMV) symptoms on plants inoculated with LMV-0-GFP, LMV-EGFP, or LMV-1/LMV-9

\begin{tabular}{|c|c|c|c|c|c|}
\hline Cultivar & Resistance status $^{\mathrm{a}}$ & Resistance gene & LMV-0-GFP & LMV-E-GFP ${ }^{c}$ & LMV-9 or LMV-1 ${ }^{\mathrm{d}}$ \\
\hline Salinas & $\mathrm{S}$ & None & $9 / 11$ & $4 / 4$ & nt \\
\hline Trocadéro & $\mathrm{S}$ & None & $7 / 7$ & $4 / 4$ & $4 / 4$ \\
\hline Vanguard & $\mathrm{S}$ & None & $11 / 11$ & $4 / 4$ & $\mathrm{nt}$ \\
\hline Alizé & $\mathrm{R}$ & $m o 1^{1}$ & $0 / 8$ & $8 / 8$ & $\mathrm{nt}$ \\
\hline Alto & $\mathrm{R}$ & $m o 1^{1}$ & $0 / 8$ & $2 / 4$ & $\mathrm{nt}$ \\
\hline Augusta & $\mathrm{R}$ & $m o 1^{l}$ & $0 / 8$ & $2 / 4$ & $\mathrm{nt}$ \\
\hline Augustus & $\mathrm{R}$ & $m o 1^{1}$ & $0 / 3$ & $3 / 3$ & $2 / 2$ \\
\hline Floricos & $\mathrm{R}$ & $m o 1^{1}$ & $0 / 8$ & $5 / 8$ & $4 / 4$ \\
\hline Floriglade & $\mathrm{R}$ & $m o 1^{l}$ & $0 / 8$ & $8 / 8$ & $4 / 4$ \\
\hline Gallega de Invierno & $\mathrm{R}$ & $m o 1^{1}$ & $0 / 8$ & $7 / 8$ & $\mathrm{nt}$ \\
\hline Krizet & $\mathrm{R}$ & $\mathrm{mol}^{1}$ & $0 / 8\left(1 / 8^{*}\right)$ & $8 / 8$ & $\mathrm{nt}$ \\
\hline Mantilia & $\mathrm{R}$ & $m o 1^{1}$ & $0 / 8$ & $4 / 4$ & $4 / 4$ \\
\hline Mirian & $\mathrm{R}$ & $\mathrm{mol}^{1}$ & $0 / 4$ & $3 / 4$ & $\mathrm{nt}$ \\
\hline Nevada & $\mathrm{R}$ & $m o 1^{1}$ & $0 / 8$ & $4 / 4$ & $\mathrm{nt}$ \\
\hline Romulus & $\mathrm{R}$ & $m o 1^{l}$ & $0 / 3$ & $3 / 3$ & $2 / 2$ \\
\hline Rossia & $\mathrm{R}$ & $m o 1^{1}$ & $0 / 8$ & $4 / 4$ & $\mathrm{nt}$ \\
\hline Sangria & $\mathrm{R}$ & $m o 1^{1}$ & $0 / 4$ & $4 / 4$ & $\mathrm{nt}$ \\
\hline Short Guzmaine & $\mathrm{R}$ & $m o 1^{l}$ & $0 / 8$ & $3 / 4$ & $4 / 4$ \\
\hline F10 line in selection & $\mathrm{R}$ & $m o 1^{1}$ & $0 / 4$ & $4 / 4$ & $\mathrm{nt}$ \\
\hline Autumn Gold & $\mathrm{R}$ & $\mathrm{mol}^{2}$ & $0 / 3$ & $0 / 3$ & $0 / 2$ \\
\hline Desert storm & $\mathrm{R}$ & $\mathrm{mol}^{2}$ & $0 / 4$ & $0 / 4$ & $0 / 4$ \\
\hline Classic & $\mathrm{R}$ & $\mathrm{mol}^{2}$ & $0 / 4$ & $0 / 4$ & $0 / 4$ \\
\hline Mojave & $\mathrm{R}$ & $m o 1^{2}$ & $0 / 3$ & $0 / 3$ & $0 / 2$ \\
\hline Salad Bibb & $\mathrm{R}$ & $m o 1^{2}$ & $0 / 3$ & $0 / 3$ & $0 / 2$ \\
\hline Salinas 88 & $\mathrm{R}$ & $\mathrm{mol}^{2}$ & $0 / 8$ & $0 / 4$ & $\mathrm{nt}$ \\
\hline Vanguard 75 & $\mathrm{R}$ & $\mathrm{mol}^{2}$ & $0 / 8$ & $0 / 4$ & $\mathrm{nt}$ \\
\hline Winterset & $\mathrm{R}$ & $\mathrm{mol}^{2}$ & $0 / 4$ & $0 / 4$ & $0 / 4$ \\
\hline
\end{tabular}

${ }^{a}$ Known resistance status: $\mathrm{S}=$ susceptible; $\mathrm{R}$ = resistant.

b Number of plants showing systemic GFP accumulation/number of LMV-0-GFP-inoculated plants. The value given corresponds to the cumulative results of Table 1. * Indicates plant negative at 8 days after inoculation but showing weak fluorescence on one leaf at 11 days after inoculation.

${ }^{c}$ Number of plants showing systemic GFP accumulation/number of LMV-E-GFP-inoculated plants. Notation performed at 8 or 9 days after inoculation. Three or four plants were used per experiment, and a value of eight inoculated plants indicates two separate experiments.

${ }^{\mathrm{d}}$ Number of plants showing LMV symptoms/number of LMV-1- or LMV-9-inoculated plants. Notation performed 6 or 8 days after inoculation, and results are not different 14 days after inoculation. $\mathrm{nt}=$ not tested. 
varieties carrying the $m o l^{2}$ resistance gene from the susceptible varieties or from those carrying the $\mathrm{mol}^{1}$ gene.

Systemic movement of LMV-0-GFP is not affected in pea. In order to evaluate whether the restriction of the movement LMV-0GFP was specific to lettuce or could be also observed in another host, the behavior of the wild type and of the tagged virus were evaluated in pea. Pea has been described as a host of LMV but a screening of pea germ plasm failed to reveal significant resistance to LMV (16). However, numerous resistance genes, including recessive ones, have been described in pea against another member of the genus Potyvirus, Pea seed-borne mosaic virus (PSbMV) (13). Eleven varieties or PI lines carrying either no resistance to PSbMV or various combinations of PSbMV resistance genes (13) were therefore inoculated with LMV-0 and LMV0 -GFP and symptoms and/or GFP accumulation monitored. The results are presented in Table 3. As can be seen, systemic movement of both the wild type and the GFP-tagged viruses occurred in all pea plants tested, irrespective of their resistance status against PSbMV.

\section{DISCUSSION}

The two closely linked or allelic genes, $\mathrm{mol}^{1}(15,28)$ and $m o l^{2}$ $(15,20)$, are currently used worldwide to protect lettuce crops against the detrimental effects of LMV infection. As is frequently observed for resistance to potyviruses $(6,9)$, these genes are recessive and do not provide immunity to viral infection by common LMV strains. These genes restrict the long-distance movement of the virus (7) or affect symptom expression and/or viral accumulation $(2,3,15,18,29)$. As a consequence of these incomplete resistance characteristics, evaluation of the resistance status cannot be performed reliably with ELISA assays (10) and thus relies on symptom observation, which may also be difficult because symptoms are affected both by environmental conditions and by lettuce varietal type $(2,3,10,29)$. However, evaluation of $\mathrm{mol}^{2}$ resistance can be simplified by the use of isolates such as LMV-1 or LMV-9 against which a complete resistance phenotype is observed. Given these difficulties or limitations, the development of a simpler and more reliable assay is highly desirable. The results presented here show that the use of the recombinant virus LMV-0-GFP expressing the GFP as a fusion with the HC-Pro provides just such a simple assay, particularly for the $\mathrm{mol}^{l}$ gene.

The ability to tag potyviral genomes by insertion of a reporter gene between the P1 and HC-Pro proteins and expression of this reporter as a translational fusion to the HC-Pro was initially demonstrated with Tobacco etch virus (TEV) (4) and later extended to several other members of the genus (17). The use of such recombinant viruses expressing GUS, bar (glufosinate resistance), or a chloroplast-targeted cytochrome $\mathrm{P} 450$ for the screening of eco- types or mutants of Arabidopsis thaliana $(11,30)$ has already been reported in the case of TEV. Similarly, recombinant TEV isolates expressing the GUS gene were used to study the mechanism of the resistance to TEV of the V20 tobacco cultivar (25).

The recombinant LMV isolates used here were developed in a similar manner $(7,31)$. These viruses showed attenuated symptoms in all lettuce hosts tested compared with the parental virus from which they were derived ( 7 ; this study), with the notable exception of their increased severity in cv. Brunia (Fig. 1C and D) for which there is currently no explanation. Although the GFP-tagged recombinants are quite stable (stability and GFP fluorescence have been observed throughout the entire life cycle of early flowering lines of lettuce), the GFP insert may sometimes be lost upon prolonged multiplication (7). The deleted viruses recovered no longer express GFP and may revert to more severe symptoms (7). Although the heterogeneous behavior of some lines observed upon recombinant virus infection during this study is most probably explained by inoculation escapes, demonstrated by the fact that homogeneous behavior was observed upon retesting, another possibility is that the susceptible plants failing to show fluorescence contained a recombinant that had lost the GFP insert. The third possibility, that the cultivars used were segregating for resistance is highly unlikely because the seeds used were from fixed commercial cultivars.

Because individual experiments reported here spanned about a 1 -year period and were performed under standard glasshouse conditions with no specific efforts to reduce season-to-season variations in environmental conditions, the behavior of the recombinant viruses in the various lettuce types does not appear strongly influenced by environmental conditions. Similar to what happens with the wild-type virus, the time needed for systemic movement of the recombinant viruses and, therefore, for the visualization of the GFP fluorescence, may however be somewhat longer during the winter months. Tests can nevertheless be performed in glasshouses without the need for controlled-environment growth chambers. The only constraint is the need for a dark room or the obligation to perform evaluations at night so that daylight does not interfere with the observation of fluorescence under UV light illumination. In these conditions, observation of GFP fluorescence is very straightforward and can be performed on any varietal type or cultivar, even by untrained personnel. In addition, the recombinant viruses used in this study are easy to confine because GFPtagging of the HC-Pro abolishes aphid transmissibility (7) as well as seed transmissibility (7; our own unpublished results with LMV-0-GFP).

There is currently no explanation for the restricted-movement behavior of LMV-0-GFP in mol varieties or of LMV-E-GFP in $m o l^{2}$-carrying varieties. Both observations are surprising because in each case, the wild-type parental isolate is able to replicate and

TABLE 3. Systemic green fluorescent protein (GFP) accumulation and Lettuce mosaic virus (LMV) symptoms on pea plants inoculated with LMV-0-GFP or LMV-0

\begin{tabular}{lccc}
\hline Cultivar & Resistance status $^{\mathrm{a}}$ & Resistance gene $^{\mathrm{b}}$ & LMV-0-GFP $^{\mathrm{c}}$ \\
\hline Scout & $\mathrm{S}$ & None & $3 / 3$ \\
Brutus & $\mathrm{S}$ & None & $3 / 3$ \\
Fjord & $\mathrm{S}$ & None & $3 / 3$ \\
Bonneville & $\mathrm{R}$ & $s b m 2$ & $3 / 3$ \\
PI 347422 & $\mathrm{R}$ & $s b m 2 / 3$ & $3 / 3$ \\
DSP & $\mathrm{R}$ & $s b m 2 / 3$ & $3 / 3$ \\
PI 347484 & $\mathrm{R}$ & $s b m 2 / 3$ & $3 / 3$ \\
PI 269774 & $\mathrm{R}$ & $s b m 1+s b m 2 / 3$ & $3 / 3$ \\
PI 269818 & $\mathrm{R}$ & $s b m 1+s b m 4+s b m 2 / 3$ & $3 / 3$ \\
PI 193586 & $\mathrm{R}$ & $3 / 3$ & $3 / 3$ \\
PI 347329 & $\mathrm{R}$ & Seggregating for $s b m 1+\operatorname{sbm} 4+\operatorname{sbm} 2 / 3$ & $3 / 3$ \\
\end{tabular}

a Known Pea seed-borne mosaic virus (PSbMV) resistance status: $\mathrm{S}=$ susceptible; $\mathrm{R}=$ resistant.

b PSbMV resistance genes present according to Olsen and Johansen (13).

c Number of plants showing systemic GFP accumulation/number of LMV-0-GFP-inoculated plants. Notation performed 11 days after inoculation.

d Number of plants showing LMV symptoms/number of LMV-0-inoculated plants. Notation performed 11 days after inoculation. 
move systemically in susceptible as well as in resistant plants $(3,18)$. This effect thus appears to be directly linked with the addition of an N-terminal translational fusion to the viral HC-Pro protein. Results with a similarly tagged LMV-E-GUS have shown that the failure to observe systemic viral movement in $\mathrm{mol}^{2}$-resistant cultivars such as 'Vanguard 75' (22) is due to a block in longdistance viral spread (7). Similarly, the very infrequent (two plants out of a total of more than 290 inoculated plants) and late observation of a few spots of LMV-0-GFP fluorescence on two resistant varieties demonstrate the ability of the recombinant to multiply and spread cell to cell in these plants and indicate that the block observed is very likely at the level of long-distance movement. In those two cases, the low amount of GFP observed was clearly different from the high and widely spread fluorescence observed in susceptible varieties (Fig. 1E), making confusion highly improbable when interpreting results.

The tagged viruses were inoculated to pea cultivars containing a range of recessive resistance genes against another member of the genus Potyvirus, PSbMV. In all plants and irrespective of their PSbMV-resistance status, the tagged viruses were able to move systemically, with a timing very similar to that of the wild-type parents. These results show that the modified accumulation pattern of the tagged viruses observed in mol-carrying plants reflects a specific interaction with the mol genes and is unlikely to represent a general property of the tagged viruses. Further evidence that the restricted movement phenotype of tagged LMV isolates in resistant lettuce cultivars may not apply to other Potyvirus-host combinations comes from results of a study showing that a resistance-breaking GUS-tagged TEV was able to move systemically in V20 resistant tobacco (25).

By allowing a more straightforward evaluation of resistance than symptom observation or lengthier ELISA assays, GFP-tagged viruses offer a new and powerful tool that should be useful in breeding and mapping projects. It is tempting to speculate on the generality of the observations reported here and on the possibility of developing recombinant viruses for the screening of genes that are difficult to assess because they afford incomplete resistance to potyviruses (9). Although only experimental work will allow the assessment of such a possibility, the results reported and results with GUS- or bar-tagged TEV $(25,30)$ indicate that this is a distinct and promising possibility.

\section{ACKNOWLEDGMENTS}

This work was partially supported by a grant from the EPR Aquitaine and by a PRFMMIP program of the French MENRT. We thank E. J. Ryder (USDA, Salinas, CA) for providing seeds for some of the varieties tested and for useful discussions and E. Johansen (Danish Institute of Agricultural Sciences, Fredericksburg, Denmark) for providing seeds of the pea lines.

\section{LITERATURE CITED}

1. Bannerot, H., Boulidard, L., Marrou, J., and Duteil, M. 1969. Etude de la tolérance au virus de la mosaïque de laitue chez la variété Gallega de invierno. Ann. Phytopathol. 1:219-226.

2. Bos, L., Huijberts, N., and Cuperus, C. 1994. Further observations on variation of lettuce mosaic virus in relation to lettuce (Lactuca sativa), and a discussion of resistance terminology. Eur. J. Plant Pathol. 100:293314.

3. Dinant, S., and Lot, H. 1992. Lettuce mosaic virus. Plant Pathol. 41:528542.

4. Dolja, V. V., McBride, H. J., and Carrington, J. C. 1992. Tagging of plant potyvirus replication and movement by insertion of $\beta$-glucuronidase into the viral polyprotein. Proc. Natl. Acad. Sci. USA 89:10208-10212.

5. Falk, B. W., and Purcifull, D. E. 1983. Development and application of an enzyme-linked immunosorbent assay (ELISA) test to index lettuce seeds for lettuce mosaic virus in Florida. Plant Dis. 67:413-416.

6. Fraser, R. S. S. 1990. The genetics of resistance to plant viruses. Annu. Rev. Phytopathol. 28:179-200.

7. German-Retana, S., Candresse, T., Alias, E., Delbos, R., and Le Gall, O. 2000. Effects of GFP or GUS tagging on the accumulation and pathogenicity of a resistance breaking LMV isolate in susceptible and resistant lettuce cultivars. Mol. Plant-Microbe Interact. 13:316-324.

8. Grogan, R. G., Welch, J. E., and Bardin, R. 1952. Common lettuce mosaic and its control by the use of mosaic-free seeds. Phytopathology 42:573-578.

9. Grumet, R., Kabelka, E., McQueen, S., Wai, T., and Humphrey, R. 2000. Characterization of sources of resistance to the watermelon strain of Papaya ringspot virus in cucumber: Allelism and co-segregation with other potyvirus resistances. Theor. Appl. Genet. 101:463-472.

10. Irwin, S. V., Kesseli, R. V., Waycott, W., Ryder, E. J., Cho, J. J., and Michelmore, R. W. 1999. Identification of PCR-based markers flanking the recessive LMV resistance gene mo1 in an intraspecific cross in lettuce. Genome 42:982-986.

11. Mahajan, S. K., Chisholm, S. T., Whitham, S. A., and Carrington, J. C. 1998. Identification and characterization of a locus (RTM1) that restricts long-distance movement of tobacco etch virus in Arabidopsis thaliana. Plant J. 14:177-186.

12. Montesclaros, L., Nicol, N., Ubalijoro, E., Leclerc-Potvin, C., Ganivet, L., Laliberté, J. F., and Fortin, M. G. 1997. Response to potyvirus infection and genetic mapping of resistance loci to potyvirus infection in Lactuca. Theor. Appl. Genet. 94:941-946.

13. Olsen, B. S., and Johansen, I. E. 2001. Nucleotide sequence and infectious cDNA clone of the L1 isolate of Pea seed-borne mosaic potyvirus. Arch. Virol. 146:15-25.

14. Pink, D. A. C., Kostova, D., and Walkey, D. G. A. 1992. Differentiation of pathotypes of lettuce mosaic virus. Plant Pathol. 41:5-12.

15. Pink, D. A. C., Lot, H., and Johnson, R. 1992. Novel pathotypes of lettuce mosaic virus-breakdown of a durable resistance. Euphytica 63: 169-174.

16. Provvidenti, R. 1973. Occurrence of Lettuce mosaic virus in Pisum sativum. Plant Dis. Rep. 57:688-690.

17. Revers, F., Le Gall, O., Candresse, T., and Maule, A. J. 1999. New advances in understanding the molecular biology of plant/potyvirus interactions. Mol. Plant-Microbe Interact. 12:367-376.

18. Revers, F., Lot, H., Souche, S., Le Gall, O., Candresse, T., and Dunez, J. 1997. Biological and molecular variability of lettuce mosaic virus isolates. Phytopathology 87:397-403.

19. Revers, F., Yang, S. J., Walter, J., Lot, H., Souche, S., LeGall, O., Candresse, T., and Dunez, J. 1997. Comparison of the complete nucleotide sequences of two isolates of lettuce mosaic virus differing in their biological properties. Virus Res. 47:167-177.

20. Ryder, E. J. 1970. Inheritance of resistance to common lettuce mosaic. J. Am. Soc. Hortic. Sci. 95:378-379.

21. Ryder, E. J. 1973. Seed transmission of lettuce mosaic virus in mosaic resistant lettuce. J. Am. Soc. Hortic. Sci. 98:610-614.

22. Ryder, E. J. 1979. Vanguard 75 lettuce. Hortscience 14:284-286.

23. Ryder, E. J. 1991. Salinas 88 lettuce. Hortscience 26:439-440.

24. Ryder, E. J., and McCreight, J. D. 1999. Lettuce. Hortscience 34:801806.

25. Schaad, M. C., and Carrington, J. C. 1996. Suppression of long-distance movement of tobacco etch virus in a nonsusceptible host. J. Virol. 70:2556-2561.

26. Shukla, D. D., Ward, C. W., and Brunt, A. A. 1994. The Potyviridae. $\mathrm{CAB}$ International, Wallingford, UK.

27. Tomlinson, J. A. 1970. Lettuce mosaic virus. No. 9 in: Descriptions of Plant Viruses. Commonw. Mycol. Inst./Assoc. Appl. Biol., Kew, England.

28. Von der Pahlen, A., and Crnko, J. 1965. El virus del mosaico de la lechuga (Marmor lactucae) en Mendoza y Buenos Aires. Rev. Invest. Agropecuarias 11:25-31.

29. Walkey, D. G. A., Ward, C. M., and Phelps, K. 1985. Studies on lettuce mosaic virus resistance in commercial lettuce cultivars. Plant Pathol. 34:545-551.

30. Whitham, S. A., Yamamoto, M. L., and Carrington, J. C. 1999. Selectable viruses and altered susceptibility mutants in Arabidopsis thaliana. Proc. Natl. Acad. Sci. USA 96:772-777.

31. Yang, S. J., Revers, F., Souche, S., Lot, H., Le Gall, O., Candresse, T., and Dunez, J. 1998. Construction of full-length cDNA clones of lettuce mosaic virus (LMV) and the effects of intron-insertion on their viability in Escherichia coli and on their infectivity to plants. Arch. Virol. 143: 2443-2451. 